\title{
RESEARCH
}

Open Access

\section{MicroRNA-27b targets CBFB to inhibit differentiation of human bone marrow mesenchymal stem cells into hypertrophic chondrocytes}

Shuang $L v^{1}$, Jinying $X u^{1}$, Lin Chen ${ }^{1,2}$, Haitao Wu ${ }^{1,3}$, Wei Feng ${ }^{4}$, Yangyang Zheng ${ }^{1}$, Pengdong $\mathrm{Li}^{1}$, Haiying Zhang ${ }^{1}$, Lihong Zhang ${ }^{1}$, Guangfan $\mathrm{Chi}^{1 *}$ (D) and Yulin $\mathrm{Li}^{1 *}$

\begin{abstract}
Background: Human bone marrow-derived mesenchymal stem cells (hBMSCs) have chondrocyte differentiation potential and are considered to be a cell source for cell-transplantation-mediated repair of cartilage defects, including those associated with osteoarthritis (OA). However, chondrocyte hypertrophic differentiation is a major obstacle for the application of hBMSCs in articular cartilage defect treatment. We have previously shown that microRNA-27b (miR-27b) inhibits hypertrophy of chondrocytes from rat knee cartilage. In this study, we investigated the role of miR-27b in chondrocyte hypertrophic differentiation of hBMSCs.

Methods: Chondrogenic marker and microRNA expression in hBMSC chondrogenic pellets were evaluated using RT-qPCR and immunohistochemistry. The hBMSCs were transfected with miR-27b before inducing differentiation. Gene and protein expression levels were analyzed using RT-qPCR and western blot. Coimmunoprecipitation was used to confirm interaction between CBFB and RUNX2. Luciferase reporter assays were used to demonstrate that $C B F B$ is a miR-27b target. Chondrogenic differentiation was evaluated in hBMSCs treated with shRNA targeting CBFB. Chondrogenic hBMSC pellets overexpressing miR-27b were implanted into cartilage lesions in model rats; therapeutic effects were assessed based on histology and immunohistochemistry.
\end{abstract}

(Continued on next page)

\footnotetext{
* Correspondence: guangfan130@jlu.edu.cn; ylli@jlu.edu.cn

'The Key Laboratory of Pathobiology, Ministry of Education, Department of

Pathology, College of Basic Medical Sciences, Jilin University, Changchun 130021, China

Full list of author information is available at the end of the article
}

(c) The Author(s). 2020 Open Access This article is licensed under a Creative Commons Attribution 4.0 International License, which permits use, sharing, adaptation, distribution and reproduction in any medium or format, as long as you give appropriate credit to the original author(s) and the source, provide a link to the Creative Commons licence, and indicate if changes were made. The images or other third party material in this article are included in the article's Creative Commons licence, unless indicated otherwise in a credit line to the material. If material is not included in the article's Creative Commons licence and your intended use is not permitted by statutory regulation or exceeds the permitted use, you will need to obtain permission directly from the copyright holder. To view a copy of this licence, visit http://creativecommons.org/licenses/by/4.0/ The Creative Commons Public Domain Dedication waiver (http://creativecommons.org/publicdomain/zero/1.0/) applies to the data made available in this article, unless otherwise stated in a credit line to the data. 
(Continued from previous page)

Results: The hBMSCs showed typical MSC differentiation potentials. During chondrogenic differentiation, collagen 2 and 10 (COL2 and COL 10), SOX9, and RUNX2 expression was upregulated. Expression of miR-140, miR-143, and miR$181 \mathrm{a}$ increased over time, whereas miR-27b and miR-221 were downregulated. Cartilage derived from hBMSC and overexpressing miR-27b exhibited higher expression of COL2 and SOX9, but lower expression of COL 10, RUNX2, and CBFB than did the control cartilage. CBFB and RUNX2 formed a complex, and CBFB was identified as a novel miR27b target. CBFB knockdown by shRNA during hBMSC chondrogenic differentiation led to significantly increased COL2 and SOX9 expression and decreased COL10 expression. Finally, miR-27b-overexpressing hBMSC chondrogenic pellets had better hyaline cartilage morphology and reduced expression of hypertrophic markers and tend to increase repair efficacy in vivo.

Conclusion: MiR-27b plays an important role in preventing hypertrophic chondrogenesis of hBMSCs by targeting CBFB and is essential for maintaining a hyaline cartilage state. This study provides new insights into the mechanism of hBMSC chondrocyte differentiation and will aid in the development of strategies for treating cartilage injury based on hBMSC transplantation.

Keywords: hBMSCs, Chondrocytes, Hypertrophic differentiation, miR-27b, CBFB, RUNX2

\section{Background}

Cartilage defects, including osteochondral lesions, are common in orthopedics and are caused by trauma, necrosis, inflammation, and degeneration $[1,2]$. Osteoarthritis (OA) is also one of the primary causes of cartilage defects; its global prevalence has been estimated to be over 250 million people [3]. Current therapies for cartilage defects include debridement, bone marrow stimulation, osteochondral grafting, arthroplasty, and autologous chondrocyte transplantation [4-6]. However, these treatments have limitations, such as small repair areas, limited cartilage sources, and microfractures that often cause fibrocartilage repair [7]. There is a clear need for more efficient approaches.

Accumulating evidence from basic and clinical studies demonstrates that beyond their multidifferentiation capabilities (e.g., osteogenesis, chondrogenesis, and adipogenesis), hBMSCs also have other beneficial effects, such as paracrine effects, antiinflammatory activities, and immunomodulatory capacities. Therefore, hBMSCs have been proposed as a source for cartilage regeneration in the treatment of cartilage injury and osteochondral lesions [8, 9]. However, hBMSCs recapitulate the endochondral differentiation of growth plate chondrocytes; moreover, cells differentiated from hBMSCs tend to be hypertrophic chondrocytes that express COL10 [10] and matrix metalloproteinase 13 (MMP13) [11] and undergo mineralization, eventually leading to endochondral bone formation in vivo. This process is controlled exquisitely by cellular interactions with the surrounding matrix, growth, and differentiation factors, as well as by other environmental factors that initiate or suppress cellular signaling pathways and transcription of specific genes in a temporospatial manner [12]. During hypertrophic differentiation, multiple factors positively modulate this process from multiple signaling pathways including TGF- $\beta$, BMP, Wnt, and Indian hedgehog $(\mathrm{IHH})$, as well as transcription factors including Runx2 and MEF2C [13, 14]. Additionally, noncoding RNAs including microRNAs (miRNAs) and long noncoding RNAs (LncRNAs) participate in and control this processing $[15,16]$. However, the underlying mechanisms by which they influence differentiation have not been fully elucidated. Hence, it is necessary to study the mechanisms underlying hypertrophic chondrocyte differentiation to help design strategies to inhibit this process and maintain the normal chondrocyte phenotype.

MicroRNAs (miRNAs) are a class of noncoding small endogenous RNAs (20-24 nucleotides in length) that either suppress translation or degrade target mRNAs through binding to their $3^{\prime}$-untranslated regions (UTRs) [17]. They play critical roles in multiple cellular processes including differentiation, development, proliferation, and tumorigenesis [18]. Generally, a single miRNA regulates multiple target genes and can have profound effects on key signaling pathways. They are also involved in chondrogenic differentiation of hBMSCs. For example, miR-29b, possibly via its target gene histone deacetylase 4 (HDAC4), promotes the hypertrophic phenotype [19]. Overexpression of miR-145 in C3H10T1/2 cells reduced SOX9 expression, thus significantly suppressing cartilage matrix synthesis in vitro [20]. Additionally, miR-495, miR92a, miR-483, miR-892b, and miR-218 have been found to play important roles in hBMSC chondrogenic differentiation [21-25]. Whether other miRNAs are involved in this process has not yet been clearly elucidated.

In a previous study, we found that in chondrocytes from knee cartilage of 1-3-day-old rat pups, miR-27b 
inhibited hypertrophy by targeting peroxisome proliferator-activated receptor $\gamma^{2}$ (PPAR 2 2) [26]. MMP13, which is a hypertrophic chondrocyte marker, is a direct target of miR-27b [27]. In a preliminary study, following induction of chondrogenic differentiation in rabbit BMSCs through high-density pellet culture for 21 days, we observed that miR-27b expression in the BMCderived chondrocytes was significantly lower than that in normal chondrocytes isolated from knee cartilage of the same donor. Therefore, we hypothesized that miR-27b may play a role in hypertrophic differentiation of hBMSC-derived chondrocytes.

Core-binding factor, $\beta$-subunit $(\mathrm{CBFB})$ is a nonDNA-binding partner of the Runt-related transcription factors1-3 (RUNX1-3). It cooperates with these factors to form DNA-protein complexes and protects them from degradation [28, 29]. CBFB is involved in skeletal development and osteoblast and chondrocyte differentiation, as well as in fracture healing [30, 31]. RUNX2 is an essential transcription factor involved in osteogenesis and chondrocyte hypertrophy. In articular cartilage, RUNX2 is extensively expressed in prehypertrophic and hypertrophic chondrocytes. RUNX2 regulates expression of COL10, SPP1, IBSP, MMP13, and VEGFA, all critical markers for hypertrophic chondrocytes [32, 33]. Thus, RUNX2 most likely functions during hBMSC chondrogenic differentiation. Therefore, it would be interesting to investigate whether downregulation of RUNX2 or/and CBFB would block or delay hypertrophic differentiation. Using the target prediction program TargetScan, we found that the 3'-UTR of CBFB mRNA, but not that of RUNX2 mRNA, has putative miR-27b-binding sites. Based on this finding, we hypothesized that $C B F B$ may be a direct target of miR-27b and that by modulating $C B F B$ expression, miR-27b may inhibit hypertrophy during hBMSC chondrogenic differentiation.

In this study, we aimed to determine the expression pattern and function of miR-27b during hBMSC chondrogenic differentiation, which are crucial for overcoming the obstacle of hypertrophic differentiation and will aid in improving the therapeutic efficacy of hBMSC-derived chondrocytes in the repair of hyaline cartilage defects or OA.

\section{Methods}

\section{Human subjects}

1 Human bone marrow aspirates were obtained from patients undergoing elective bone surgery $(n=5$ donors, age $30-60$ years, 3 men, 2 women). All participants were informed regarding the nature of this study and gave written informed consent. The study was approved by the Ethics Committee of The First Hospital of Jilin University (approval 2017-342).

\section{Isolation and culture of hBMSCs}

Twenty-milliliter aliquots of bone marrow suspension were centrifugated and washed twice with PBS. Cells were resuspended in DMEM/F12 (1:1, HyClone, USA) containing $10 \%$ fetal bovine serum (FBS; Ausbian, Australia) and $10 \mathrm{ng} / \mathrm{ml}$ basic fibroblast growth factor (bFGF; Active Bioscience, Germany) and seeded onto culture dishes. Cultures were maintained in an incubator with $5 \% \mathrm{CO}_{2}$ at $37^{\circ} \mathrm{C}$. Once cells reached $>90 \%$ confluence (passage 0 ), they were passaged using $0.25 \%$ trypsin/ethylenediaminetetraacetic acid (EDTA). Cells from passage 4 were used in the experiments. Samples from the five individuals were processed independently.

\section{Flow cytometry}

In brief, hBMSCs were trypsinized and washed with phosphate-buffered saline (PBS) twice. The cell concentration was adjusted to $0.5 \times 10^{5}$ per tube. Cells were incubated with FITC-labeled antibodies against CD34, CD44, CD45, CD73, CD90, and CD105 (Bioscience, USA) at $4{ }^{\circ} \mathrm{C}$ in the dark for $30 \mathrm{~min}$. Cells were washed with PBS twice and centrifuged. The cells were fixed with $4 \%$ paraformaldehyde and analyzed in a FACSCanto $^{\text {tw }}$ II Flow Cytometer (BD Biosciences).

\section{Chondrogenic differentiation induction}

Cells $\left(2.5 \times 10^{5}\right)$ were resuspended in $0.5 \mathrm{ml}$ of cartilage-inducing medium, high-glucose DMEM (HyClone), $100 \mathrm{nM}$ dexamethasone, $100 \mu \mathrm{g} / \mathrm{ml}$ sodium pyruvate, $25 \mathrm{mg} / \mathrm{ml}$ vitamin $\mathrm{C}, 40 \mathrm{mg} / \mathrm{ml} \mathrm{D}$-valine, 10 $\mathrm{ng} / \mathrm{ml}$ transforming growth factor $\beta 3$ (TGF- $\beta 3$ ), and $1 \%$ ITS (Sigma-Aldrich), and pelleted. The pellet was incubated in an incubator with $5 \% \mathrm{CO}_{2}$ at $37^{\circ} \mathrm{C}$. The medium was refreshed every 2 days and chondrogenic pellets were harvested after 7,14 , and 21 days. The harvested pellets were fixed with $4 \%$ paraformaldehyde for $72 \mathrm{~h}$, embedded in paraffin, sectioned at 5$\mu \mathrm{m}$ thickness, and stained with Alcian blue (Cyagen, China) and Safranin O (Sigma-Aldrich). Tissue organization and extracellular matrix deposition were analyzed by microscopy (Olympus, Japan).

\section{Vector construction and packaging}

The pLV3 shuttle (Jima, China) and packaging (pGag/ Pol, pRev, and pVSV-G) plasmids were transfected into 293T cells (Cell Bank of the Chinese Academy of Sciences, China) using RNAi-Mate (Jima, China). The sequences harbored in the pLV3 shuttle plasmid were as follows: miR-27b (full-length $5^{\prime}$-ACCUCUCUAA CAAGGUGCAGAGCUUAGCUGAUUGGUGAACAG UGAUUGGUUUCCGCUUUGUUCACAGUGGCUAA GUUCUGCACCYGAAGAGAAGGUG-3'); shRNACBFB (GGAGGCAAGAAGACAACAAGA). Cells were cultured in DMEM/F12 (HyClone) medium with $10 \%$ 
FBS (Ausbian) for $48 \mathrm{~h}$. The lentivirus-containing medium was collected, and the virus was concentrated using Centricon centrifugal filters (Beckman, USA).

\section{Lentiviral transduction and selection}

For lentiviral transduction, hBMSCs prior to differentiation were seeded in 6-well culture plates at $2 \times 10^{5}$ cells/well and cultured in 10\% FBS (Ausbian)-containing DMEM/F12 (HyClone) for $24 \mathrm{~h}$. Then, the cells were transfected with lentiviral at a multiplicity of infection of 50 in medium containing $5 \mu \mathrm{g} / \mathrm{ml}$ polybrene for $12 \mathrm{~h}$, after which the medium was refreshed. Puromycin $(1 \mu \mathrm{g} /$ $\mathrm{ml}$ ) was added to the medium for 3 days to select stably transfected cells.

\section{Mimic-mediated overexpression of miR-27b and siRNA- mediated knockdown of $\beta$-catenin in hBMSCs}

Cells were seeded in 6-well plates at $2 \times 10^{5} /$ well and cultured for $24 \mathrm{~h}$. Pre-miR-27b mimic (Ambion, USA) was delivered into the cells using DharmaFECT1 (Life Technologies, Germany) following the manufacturer's protocol. Briefly, mimics $(100 \mathrm{nM})$ were mixed with the transfection agent in Opti-MEM (Invitrogen, USA), incubated for $20 \mathrm{~min}$, and added to serum-free medium. As controls, cells were transfected with premiRNA precursor mimics (Ambion, USA) or treated with transfection reagents alone. Twenty-four hours later, the medium was replaced with cartilageinducing medium. Cells were cultured for another 48 $\mathrm{h}$ for RT-qPCR and western blotting. The transfection procedure of siRNA is in line with that of mimics.

\section{RT-qPCR}

RNA was extracted from cells using TRIzol (Invitrogen, USA). Expression levels of miRNAs were measured with the All-in-One miRNA RT-qPCR Detection Kit (GeneCopoeia, USA). U6 was used as a reference gene for normalization. Samples were analyzed on an ABI 7300 qPCR instrument (Applied Biosystems, USA). RT-qPCR reactions for COL2, COL10, CBFB, MMP13, PPAR , and SOX9 were run using TransStart Green qPCR SuperMix (TransGen, China) in the ABI7300 qPCR instrument. PCR primer sequences are listed in Table 1. Gene expression was normalized to that of GAPDH and quantified using the $2^{-\Delta \Delta \mathrm{Ct}}$ method.

\section{Western blotting}

Cells were harvested and total proteins were extracted using RIPA buffer (Beyotime Biotechnology, China). Protein concentrations were assessed using a Pierce BCA Assay kit (Beyotime Biotechnology). Total protein $(30 \mu \mathrm{g})$ was loaded onto $10 \%$ sodium dodecyl sulfate polyacrylamide gels, electrophoresed, and transferred to a polyvinylidene fluoride membrane. The membrane was blotted with antibodies against COL2 (1:1000, Abcam, USA), SOX9 (1:2000, Sigma-Aldrich), RUNX2 (1:500, Sigma-Aldrich), MMP13 (1:1000, Abcam), CBFB (1: 1000, Santa Cruz Biotechnology, USA), COL10 (1:2000, Abcam), $\beta$-catenin (1:1000, CST, USA), PPARY (1:1000, CST, USA), and GADPH (1:2000, TransGen) at $4{ }^{\circ} \mathrm{C}$ overnight, followed by incubation with peroxidaseconjugated secondary anti-mouse or anti-rabbit IgG antibody (1:2000, TransGen) at room temperature for $60 \mathrm{~min}$. Signals were detected by chemiluminescence using the ECL-Plus detection system (TransGen).

Table 1 Primers of qPCR

\begin{tabular}{lll}
\hline Gene & Forward primers (5' to 3') $^{\prime}$ & Reverse primers (3' to 5') \\
\hline CBFB & GGCGCGGCTGAGGGCGGGAGA & CGTTAAGTGAGCACAGCTTATG \\
COL2 & CGTCCAGATGACCTTCCTACG & TGAGCAGGGCCTTCTTGAG \\
COL10 & GGCAGAGGAAGCTTCAGAAA & AAGGGTATTTGTGGCAGCATA \\
GAPDH & CCATGTTCGTCATGGGTGTGA & CATGGACTGTGGTCATGAGT \\
MMP13 & GAGTGGACTCACTGTTGGTC GCAAGAGTCACAGGATGGTAG \\
PPARY & CTGGCCTCCCTGATGAATAA & CGCAGGTTTTGAGGAACTC \\
RUNX2 & TTACTGTCATGGCGGGTAAC & AGGTGAAACTCTTGCCTCGT \\
SOX9 & GTACCCGCACTTGCACAAC & TCTCGCTCTCGTTCAGAAGTC \\
hsa-mir-27b & HmiRQP0361GeneCopoeia, China & \\
hsa-mir-140 & HmiRQP0182GeneCopoeia, China & \\
hsa-mir-143 & HmiRQP0188GeneCopoeia, China & \\
hsa-mir-145 & HmiRQP0191GeneCopoeia, China & \\
hsa-mir-181a & HmiRQP0231GeneCopoeia, China & \\
hsa-mir-221 & HmiRQP0338GeneCopoeia, China & \\
\hline
\end{tabular}


Protein bands were semi-quantified based on three independent experiments by densitometry using Bandscan 5.0 (Glyko Biomedical, USA) software. GAPDH was used as a loading control.

\section{Luciferase reporter assay}

The 3 '-UTR of $C B F B$ and point mutations were PCRamplified with primers flanked by XhoI and NotI restriction sites. The fragment was cloned into the pSICHECK2 vector (Promega, USA) downstream of the Renilla luciferase gene in sense and antisense orientations. Chondrocytes were seeded into 96-well plates and co-transfected with the luciferase reporter constructs and $75 \mathrm{nM}$ pre-miR-27b precursor, using DharmaFECT1. Luminescence was measured $48 \mathrm{~h}$ post transfection using the Dual-Glo Luciferase Assay System (Promega).

\section{Coimmunoprecipitation (co-IP)}

Chondrogenic differentiation was induced as described above. Cells were washed with PBS, harvested from the culture dishes, and centrifuged. Cells were lysed in icecold IP Lysis/Wash Buffer (TransGen) containing protease inhibitors. Part of the lysate was used as an input control for precipitation, while the remainder was washed with TBST (Tris $10 \mathrm{mM}, \mathrm{NaCl} 150 \mathrm{mM}$, Tween$201 \%, \mathrm{pH}$ 7.5) three times and used for co-IP with $2 \mu \mathrm{g}$ anti-CBFB antibody $(1: 1000$, CST) or anti-RUNX2 antibody (1:500, CST). After $3 \mathrm{~h}$ of incubation, Protein G Sepharose was added and the samples were incubated overnight at $4{ }^{\circ} \mathrm{C}$, then centrifuged at $12,000 \times g$ for $1 \mathrm{~min}$. The precipitates were rinsed with IP buffer $(0.5 \% \mathrm{NP}-40$, Tris $20 \mathrm{mM}, \mathrm{pH} 8.0,150 \mathrm{mM} \mathrm{NaCl}$ ) four times to remove non-specific binding molecules. IgG was used as a $\mathrm{NC}$ for precipitation. Relative expression levels of CBFB and RUNX2 in the precipitates were assessed by western blot.

\section{Articular cartilage defect rat model and cartilage pellet transplantation}

Animal surgeries were performed according to protocols approved by the Jilin University Institutional Animal Care and Use Committee and following guidelines for the care and use of laboratory animals (approval 2019064). Eighteen specific pathogen-free male SpragueDawley rats (2-3 months of age, 200-250 g) were randomly allocated into three groups: a NC group, a miR27b-SC-LV (miR-27b-scramble-LV) group, and a miR27b-LV group ( $n=6$ in each group). NC group cells were treated with transfection reagents alone. Transfected hBMSCs were induced to differentiate in culture for 14 days. Then, chondrogenic pellets were harvested for transplantation in vivo.
The right hind limb of each animal was used for defect preparation and treatment. Rats were anesthetized by intraperitoneal injection of $40 \mathrm{mg} / \mathrm{kg}$ sodium pentobarbital. After shaving and disinfection, the right knee joint was exposed through a medial parapatellar approach. The patella was dislocated laterally, without patellar eversion. A defect (1.5- $\mathrm{mm}$ diameter and $1.5-\mathrm{mm}$ depth) was created in the trochlear groove of the femur cartilage using a dental drill. Debris was removed from the defect using a curette and irrigation. Then, a chondrogenic pellet was implanted in the lesion, the patella was physically relocated, and the joint capsule, subcutaneous tissue, and skin were closed with sutures. All rats were returned to their cages after surgery and allowed to move freely. During the experiment, cyclosporine (Novartis, Switzerland) at a dose of $10 \mathrm{mg} / \mathrm{kg}$ was intraperitoneally administered on days $-2,-1,0,1,4,7$, and 10 [34]. At 4 weeks post transplantation, rats were sacrificed and their femurs excised and fixed using 10\% formalin for histological examinations.

\section{Histochemistry and immunohistochemistry}

Femoral samples were fixed in $10 \%$ buffered formalin, decalcified in $10 \%$ buffered EDTA (pH 7.4, SigmaAldrich), embedded in paraffin, and sectioned at $5-\mu \mathrm{m}$ thickness. Hematoxylin and eosin (HE) staining was employed to evaluate the histological structure, and both toluidine blue and Safranin $O$ were utilized to visualize proteoglycans and glycosaminoglycan (GAG) deposition [35]. For immunohistochemistry (IHC), after deparaffinization, rehydration, and several washes with PBS, sections were stained using the S-100 Protein Detection Kit (Fuzhou Maixin Biotech, China). Then, the sections were incubated with antibodies against COL1 (1:200, Abcam), COL2 (1:100, Abcam), SOX9 (1:200, Sigma-Aldrich), COL10 (1:200, Abcam), anti-human-nuclei antibody (1: 1000, CST), osteopontin (OPN) (1:100, Abcam), and osteocalcin (OCN) (1:100, Bio-Techne, China) stained with 3,3-diaminobenzindine and counterstained with hematoxylin. The integrated optical density was semiquantitated using ImageJ 1.52 software (Media Cybernetics, USA). The stained histological samples were imaged and analyzed to determine the extent of cartilage repair. Cartilage repair was graded blindly by two observers using the O'Driscoll score [36].

\section{Statistical analysis}

All data are presented as means \pm standard deviations from at least three independent experiments. Statistical analyses were performed using GraphPad Prism 7.0 software. Means of two groups were compared using independent-samples $t$-tests, and means of multiple groups were compared by one-way analyses of variance. For histological scoring of cartilage repair in vivo, the 
nonparametric Mann-Whitney $U$ test was used to establish the statistical significance of the graded samples across independent observations of the repaired tissue made by two different observers. $P<0.05$ was considered statistically significant.

\section{Results}

\section{Cultured hBMSCs expressing typical MSC markers}

First, we evaluated whether the cultured cells were hBMSCs. The cultured cells showed the typical spindle-shaped morphology of hBMSCs (Fig. 1a). Flow cytometry indicated that the cells were positive for CD44 (93.25\%), CD73 (99.92\%), CD90 (98.27\%), and CD105 (99\%), but negative for CD34 (0.16\%) and CD45 (0.85\%) (Fig. 1b). These results showed that the isolated and cultured cells exhibited typical MSC morphology and markers expression.

\section{Hypertrophic chondrocyte differentiation of cultured hBMSCs occurs after 2 weeks of induction}

Hypertrophic changes reportedly occur after chondrogenic induction of hBMSCs for 2 weeks [37]. We induced chondrogenic differentiation by the pellet culture method for 3 weeks and evaluated the expression of chondrogenic and hypertrophic markers at $0,7,14$, and 21 days to determine the timing of hypertrophy.

We used RT-qPCR to detect the mRNA levels of COL2, SOX9, COL10, RUNX2, and CBFB in cartilage pellets at $0,7,14$, and 21 days. COL2 $(P<0.05)$ and SOX9 $(P<0.05) \quad$ (hyaline cartilage markers) mRNA levels significantly increased on day 7 over those on day 0 . In addition, the mRNA expression of the hypertrophy markers COL10 $(P<0.001)$ and RUNX2 $(P<0.001)$ significantly increased on day 14 (Fig. 2a). Western blot analysis of the hBMSC-derived cartilage pellets revealed that the expression of COL2 $(P<$ $0.001)$ and SOX9 $(P<0.05)$ was markedly increased on day 7 , and the levels of COL10 $(P<0.001)$ and RUNX2 $(P<0.001)$ were significantly increased on day 14 (Fig. 2b, c). In addition, from IHC analysis, we found that in comparison with pellets on day 7 , after 14-day and 21-day induction, COL2 $(\mathrm{P}<0.05)$, SOX9 $(\mathrm{P}<0.001)$, and COL10 $(\mathrm{P}<0.001)$ expression increased respectively (Fig. $2 \mathrm{~d}$, e), suggesting that hBMSCs do tend to acquire a hypertrophic phenotype after chondrogenic induction in culture from day 14 .

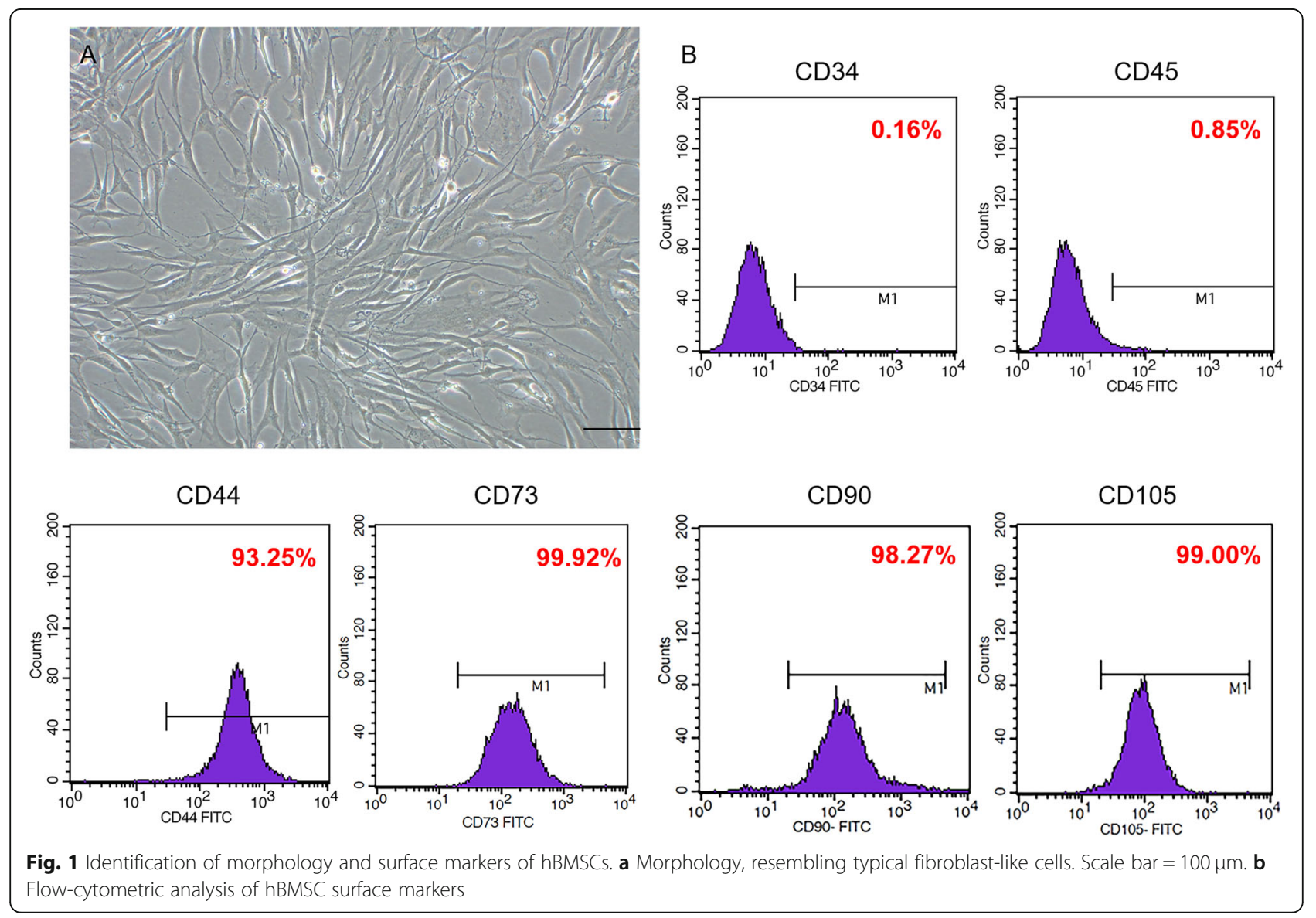




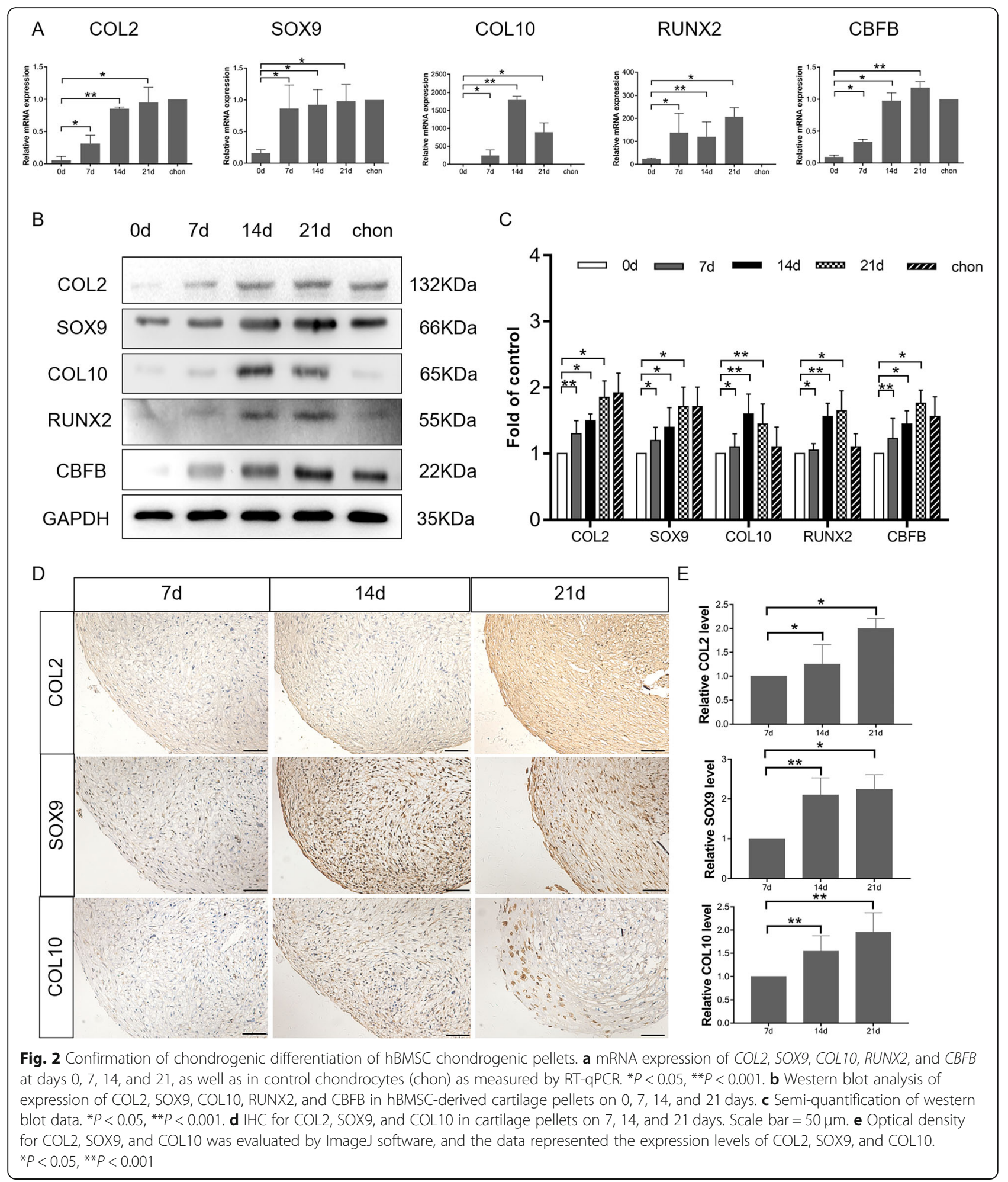

miR-27b expression is downregulated during hypertrophic chondrocyte differentiation

Certain miRNAs (e.g., miR-140, miR-143, and miR-145) have dramatically altered expression and play critical roles during chondrogenic differentiation of hBMSCs
[20, 38-40]. Therefore, we evaluated their expression during chondrogenic differentiation. The expression of miR-140 $(P<0.05)$, miR-143 $(P<0.05)$, and miR-181a $(P<0.05)$ gradually increased on days 7 and 14 relative to their levels on day 0 . The expression of miR-140 $(P<$ 
$0.05)$ and miR-143 $(P<0.05)$ was significantly higher in hBMSC-derived cartilage pellets than in normal chondrocytes. In contrast, miR-181a $(P<0.05)$ was expressed at a lower level in cartilage pellets than in normal chondrocytes. The miR-145 $(P<0.05)$ level tended to slightly decrease over time, but was higher in cartilage pellets than in normal chondrocytes. Both miR-27b $(P<0.05)$ and miR-221 $(P<0.05)$ had significantly decreased expression on day 14 , but were consistently expressed at lower levels in cartilage pellets than in normal chondrocytes (Fig. 3). These results suggested that miR-143, miR-145, and miR-181a may be involved in hBMSC hypertrophic chondrocyte differentiation, whereas miR$27 \mathrm{~b}$ and miR-221 may negatively regulate this process.

\section{Overexpression of miR-27b inhibits hypertrophic chondrocyte differentiation of hBMSCs}

We previously found that miR-27b expression was inversely correlated with chondrocyte hypertrophic differentiation in postnatal rat knee articular cartilage [26]. Therefore, we next focused on its during hBMSC chondrogenic differentiation in culture. The hBMSCs were incubated with a chemically synthesized miR-27b mimic for 3 days to exogenously enhance miR-27b expression. COL2 $(P<0.001)$ and SOX9 $(P<0.001)$ mRNA levels were significantly upregulated, whereas the mRNA expression levels of
RUNX2 $(P<0.001)$, CBFB $(P<0.001)$, MMP13 $(P<$ $0.05)$, and PPAR $\gamma(P<0.05)$ were significantly downregulated (Fig. 4a). In accordance, western blot analysis of the hBMSC-derived cartilage pellets revealed that the protein expression of COL2 $(P<0.001)$ and SOX9 $(P<0.001)$ was markedly increased in the miR27b-overexpressing hBMSCs relative to NC, whereas the levels of RUNX2 $(P<0.05)$, MMP13 $(P<0.05)$, PPAR $\gamma(P<0.001)$, and CBFB $(P<0.05)$ were significantly decreased (Fig. 4b, c). This finding supported our hypothesis that miR-27b may be promoting chondrogenesis and inhibiting hypertrophy.

We then utilized a miR-27b-LV to enhance miR-27b expression in hBMSCs and induced chondrogenic differentiation by pellet culture. RT-qPCR confirmed that after transfection, miR-27b expression was upregulated 4.7 -fold $(P<0.001)$ and was maintained at a high level for up to 14 days (Fig. $4 \mathrm{~d}$, e). Cartilage pellets after 14day induction were fixed and subjected to IHC for COL2, SOX9, and COL10 (Fig. 4f, g). We found that overexpression of miR-27b promoted the expression of COL2 $(P<0.05)$ and SOX9 $(\mathrm{P}<0.05)$ when compared with the levels in cells treated with miR-27b-LV, miR27b-SC-LV, or NC. In contrast, COL10 $(P<0.05)$ expression declined slightly in the miR-27b-overexpression group relative to that in the $\mathrm{NC}$ groups. These results suggested that overexpression of miR-27b in hBMSCs

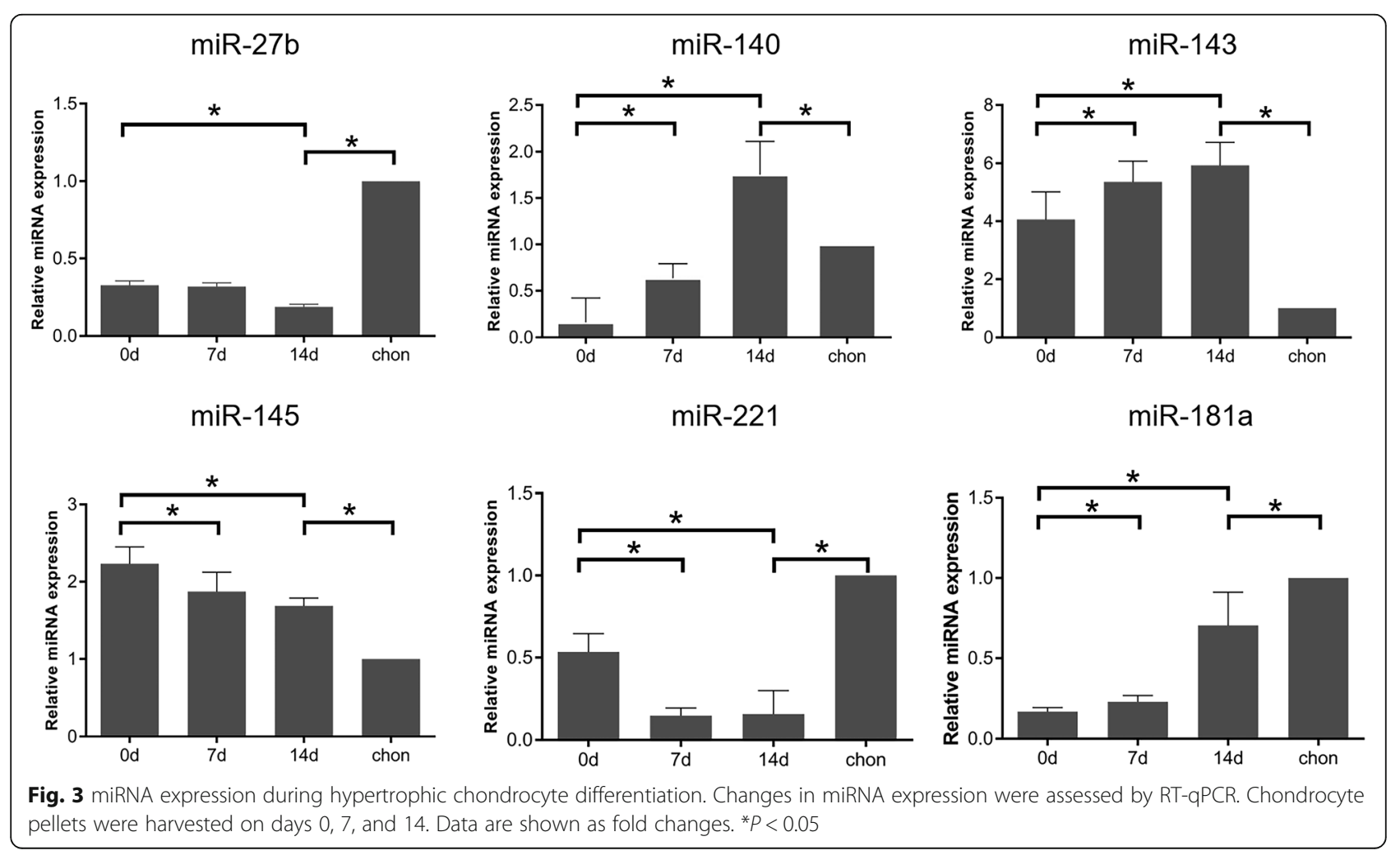




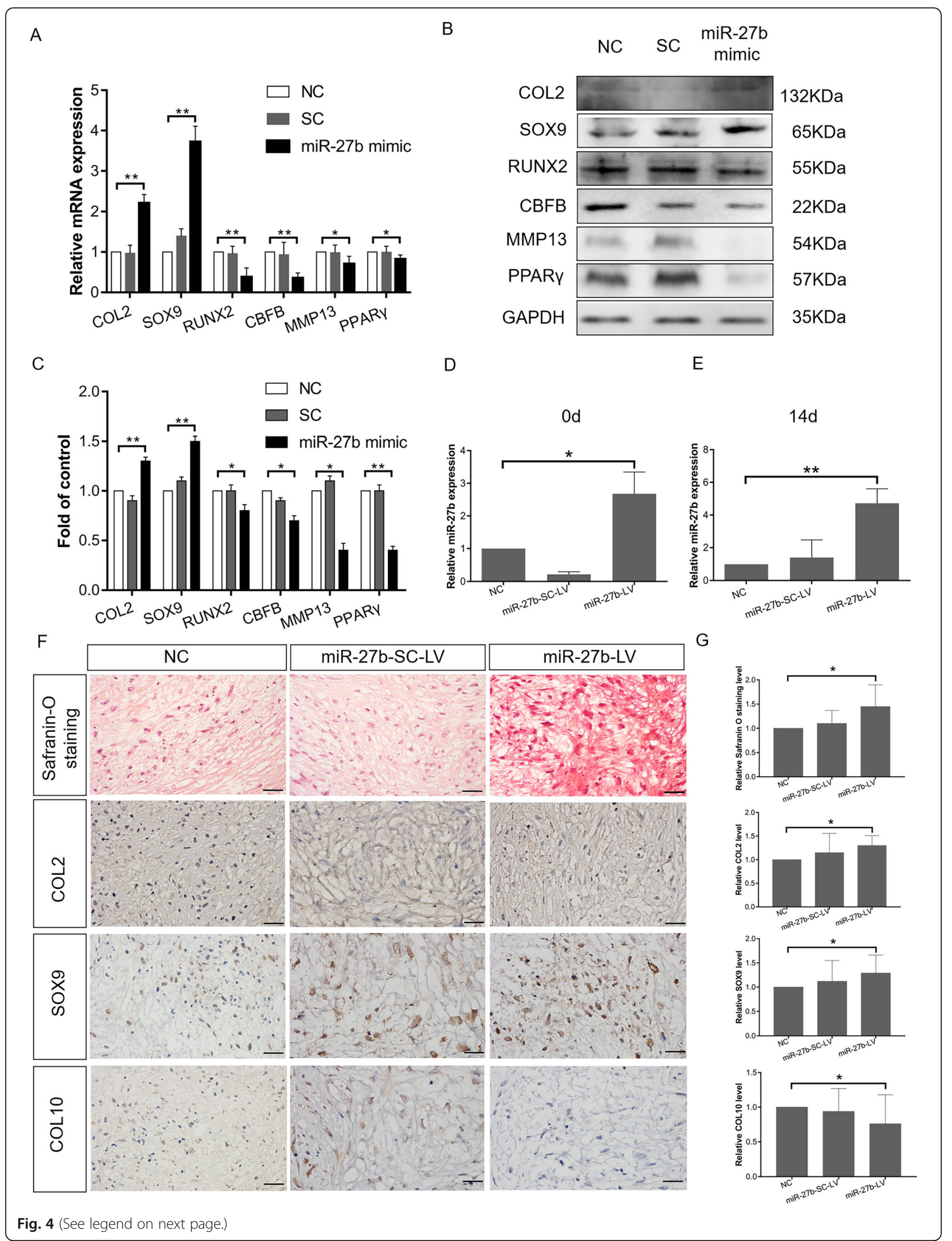


(See figure on previous page.)

Fig. 4 miR-27b overexpression inhibits hypertrophic differentiation of hBMSC-derived chondrocytes. a Expression of COL2, SOX9, RUNX2, CBFB, MMP13, and PPARy upon overexpression of miR-27b mimic in hBMSCs as measured by RT-qPCR on day 3. ${ }^{*} P<0.05$, ${ }^{* *} P<0.001$. $\mathbf{b}$ Western blot measurement of expression of COL2, SOX9, RUNX2, CBFB, MMP13, and PPARY expression in miR-27b-overexpressing hBMSCs compared to NC on day 3 after transfection. $\mathbf{c}$ Semi-quantification of western blot data. ${ }^{*} P<0.05,{ }^{* *} P<0.001$. $\mathbf{d}$ miR-27b expression on day 0 after miR-27b-LV and miR-27b-SC-LV transfection followed by chondrogenic induction, as measured by RT-qPCR. ${ }^{*} P<0.05$. e miR-27b expression on day 14 after miR27b-LV and miR-27b-SC-LV transfection followed by chondrogenic induction for 14 days, as measured by RT-qPCR. ${ }^{* *} P<0.001$. $\mathbf{f}$ Safranin $O$ staining and IHC for COL2, SOX9, and COL10 in cartilage pellets on day 14. Scale bar $=20 \mu \mathrm{m}$. g Safranin O staining was performed to measure GAGs. Optical density for GAGs was evaluated by ImageJ software, and the data represented GAG content. Optical density for COL2, SOX9, and COL10 was evaluated by ImageJ software, and the data represented the expression levels of COL2, SOX9, and COL10. ${ }^{*} P<0.05$

may effectively inhibit hypertrophic differentiation after chondrogenic induction in culture.

miR-27b inhibits hBMSC hypertrophic chondrocyte differentiation by regulating CBFB and possibly upregulates SOX9 expression by stimulating $\beta$-catenin expression

RUNX2 is an essential transcription factor for skeletal development that plays an important role in chondrocyte hypertrophy [14]. CBFB is a co-transcription factor of RUNX2; they heterodimerize to enhance DNA binding and stability of RUNX2 and inhibit its ubiquitination [41]. Co-IP was used to verify that during chondrogenic differentiation of hBMSCs, CBFB directly interacts with RUNX2 to form a complex. When total lysates of hBMSC cartilage pellets were immunoprecipitated with CBFB antibody, RUNX2 expression was detected by western blot. Conversely, when the same total lysates were immunoprecipitated with RUNX2 antibody, CBFB was present (Fig. 5a). These results demonstrated that during hBMSC chondrogenic differentiation, RUNX2 functions may rely on heterodimerization with CBFB.

We then utilized shRNA to silence $C B F B$ (Fig. 5b, c). Fourteen days after shRNA transfection, COL10 $(P<$ $0.05)$ and RUNX2 $(P<0.001)$ mRNA expression was significantly downregulated, whereas SOX9 $(P<0.05)$ and COL2 $(P<0.05)$ mRNA levels were significantly upregulated. Consistent with the mRNA data, western blotting showed that after shRNA treatment, the protein levels of RUNX2 $(P<0.001)$ and COL10 $(P<0.05)$ were significantly downregulated, with SOX9 $(P<0.001)$ protein levels significantly increased relative to the levels in $\mathrm{NC}$ pellets (Fig. 5c, d). Furthermore, IHC revealed that, in line with the western blot results, $C B F B$-silenced pellets had stronger expression of COL2 $(P<0.05)$ and SOX9 $(P<0.05)$ and weaker expression of COL10 $(P<0.05)$ than NC pellets (Fig. 5e, f). These results showed that blocking CBFB expression effectively inhibited hypertrophic chondrocyte differentiation of hBMSCs.

SOX9 is a negative regulator of chondrocyte hypertrophic differentiation [42], and the $\beta$-catenin pathway is involved in controlling SOX9 expression [43]. Thus, we examined whether miR-27b enhanced SOX9 expression through the $\beta$-catenin pathway. After miR-27b mimic treatment, SOX9 $(P<0.001)$ and $\beta$-catenin $(P<0.05)$ protein levels were both upregulated (Additional file 2: Fig. S2A and B). During chondrogenic differentiation, we treated all groups of hBMSCs with miR-27b mimics and simultaneously transfected with siRNA of $\beta$-catenin (si- $\beta$-catenin) or si-Scramble (si-SC). We found that after $\beta$-catenin interfering, the $\beta$-catenin $(P<0.05)$ and SOX9 $(P<0.001)$ protein levels were significantly downregulated (Additional file 2: Fig. S2C and D). These results suggested that miR-27b promotes chondrogenic differentiation and represses hypertrophy of hBMSCs, possibly through stimulating SOX9 expression via $\beta$ catenin pathway activation.

\section{CBFB is a novel target gene of $\mathrm{miR}-27 \mathrm{~b}$}

Target prediction using two different tools [TargetScan (www.targetscan.org) and miRDB (www.microrna.org)] suggested that $C B F B$ is a potential target of miR-27b (Additional file 3). In addition, it showed that the binding sites for miR-27b are evolutionarily conserved between humans and rats. CBFB is predicted to be targeted by miR-27b-3p in miRDB (Fig. 6a). Using RTqPCR and western blotting, we found that overexpression of miR-27b resulted in significantly decreased mRNA and protein levels of CBFB (Fig. $4 a-c$ ). In a luciferase reporter assay, CBFB-luciferase activity was significantly reduced in the presence of pre-miR-27b mimic $(P<0.001)$, whereas there was no significant effect when $C B F B$ carried a point mutation (Fig. 6b). This demonstrated that $C B F B$ was a target of miR-27b, and $\mathrm{miR}-27 \mathrm{~b}$ can directly bind to the 3 '-UTR region of $C B F B$ mRNA and downregulate its expression.

\section{Overexpression of miR-27b in hBMSCs inhibits their hypertrophic differentiation and enhances their cartilage defect repair efficacy in vivo}

To investigate miR-27b inhibition of hBMSC hypertrophic differentiation in a cartilage defect environment in vivo, we established a full-thickness articular cartilage defect in rats and transplanted cartilage pellets induced from hBMSCs. Three experimental groups were included: a NC group, a miR-27b-SC-LV group, and a 


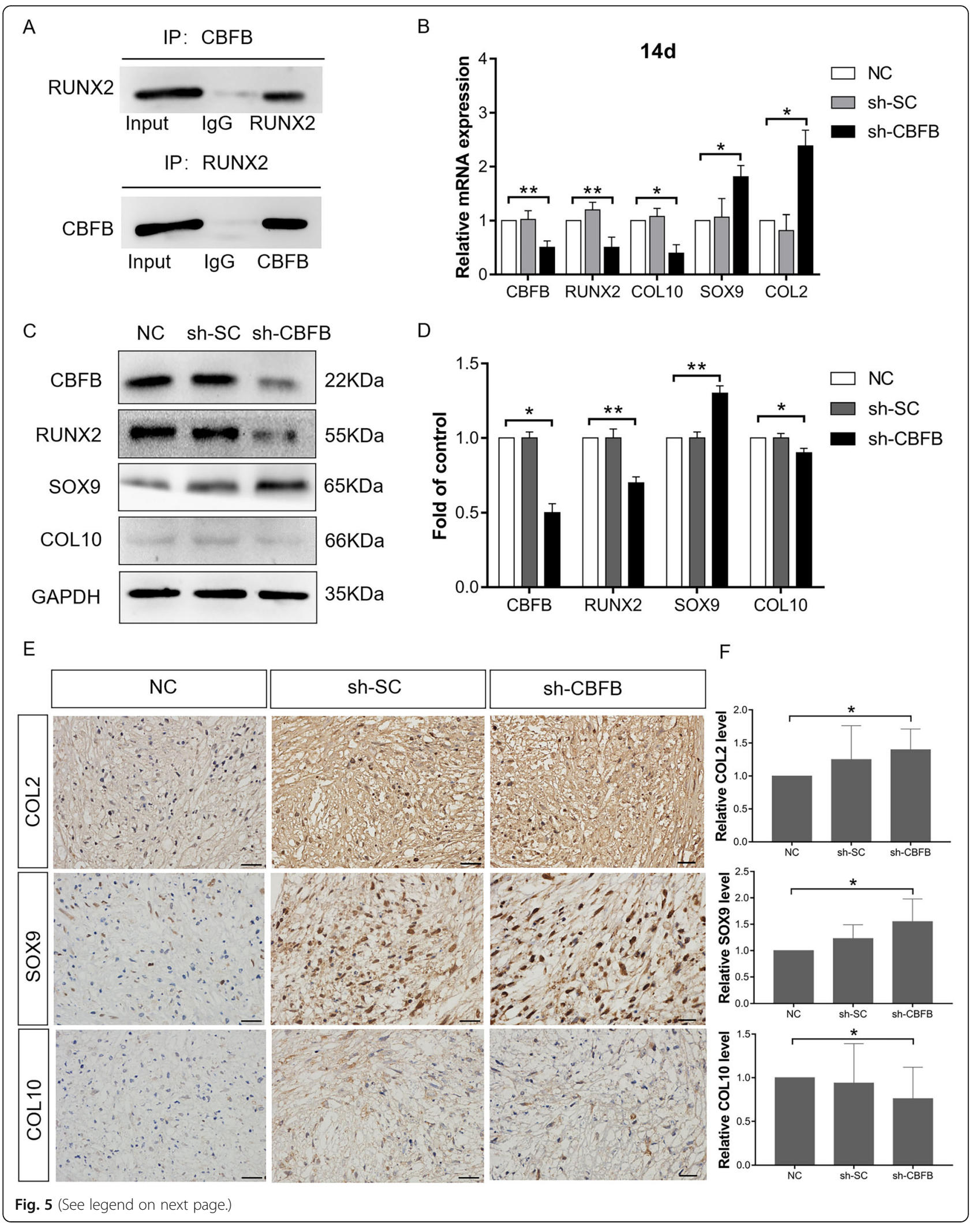


(See figure on previous page.)

Fig. 5 miR-27b inhibits hypertrophic differentiation of hBMSC-derived chondrocytes and upregulates SOX9 expression. a Protein levels of CBFB and RUNX2 in coimmunoprecipitates. b After sh-CBFB and sh-SC transfection, on 14 days mRNA expression of CBFB, RUNX2, COL10, SOX9, and COL2 as measured by RT-qPCR. ${ }^{*} P<0.05$, ${ }^{*} P<0.001$. c Protein levels of CBFB, RUNX2, SOX9, and COL10 as measured by western blot. $\mathbf{d}$ Semiquantification of western blot data. ${ }^{*} P<0.05$, ${ }^{* *} P<0.001$. e $\mathrm{IHC}$ staining of COL2, SOX9, and COL10 expression in chondrogenic pellets transfected with NC, sh-SC, and sh-CBFB. Scale bar $=20 \mu \mathrm{m}$. f Measurement of optical density for COL 2, SOX9, and COL10 was evaluated by ImageJ software, and the data represented the expression levels of COL 2, SOX9, and COL10. ${ }^{*} P<0.05$

miR-27b-LV group. Femoral samples were subjected to histological analysis using Toluidine blue and Safranin O staining (Fig. 7a, b), as well as IHC (Fig. 7c, d). Histologically, in the miR-27b-LV implants, the neogenerated tissue had a smoother surface, showed better integration with the surrounding normal cartilage, and maintained a relatively intact cartilage structure, with welldifferentiated chondrocytes surrounded by abundant matrix proteoglycan. In contrast, in the miR-27b-SC-LV and NC groups, we observed nearly complete cartilage destruction, including severe fibrillation, obvious proteoglycan loss, and fewer chondrocyte clusters. IHC revealed strong COL2 expression $(P<0.05)$, but low expression of COL1 $(P<0.05)$, a fibrous chondrocyte marker, in the miR-27b-LV group. In contrast, in the $\mathrm{NC}$ and miR-27b-SC-LV groups, COL1 $(P<0.05)$ and COL10 $(P<0.001)$ expression was higher than that in the miR-27b-LV group. Finally, human cells in the cartilage lesion site were detected using an anti-human-nuclei antibody, showing that human cells localized within the implant site and in neogenerated tissue. Using a wellestablished histological grading scale (O'Driscoll score) [36], we evaluated the degree of articular cartilage repair among the different animal groups. We found that the histological grading of the miR-27b-LV group was significantly higher than that of the $\mathrm{NC}$ group and slightly higher than that of the miR-27b-SC-LV group without significant difference (Fig. 7e). Furthermore, immunohistochemical staining for osteopontin (OPN) and osteocalcin $(\mathrm{OCN})$ showed that within the area adjacent to the hypertrophic zone of cartilage and subchondral bone, compared with the miR-27b-SC-LV group, the miR-27bLV group exhibited less number of hypertrophic chondrocytes and bone marrow cavity structures forming, and more weakly positive OPN and OCN reactions, suggesting that miR-27b certainly tend to alleviate hypertrophic differentiation of hBMSC-derived chondrocytes and endochondral bone formation in vivo (Additional file 4: Fig S4). Collectively, these results demonstrated that ectopic expression of miR-27b in hBMSCs

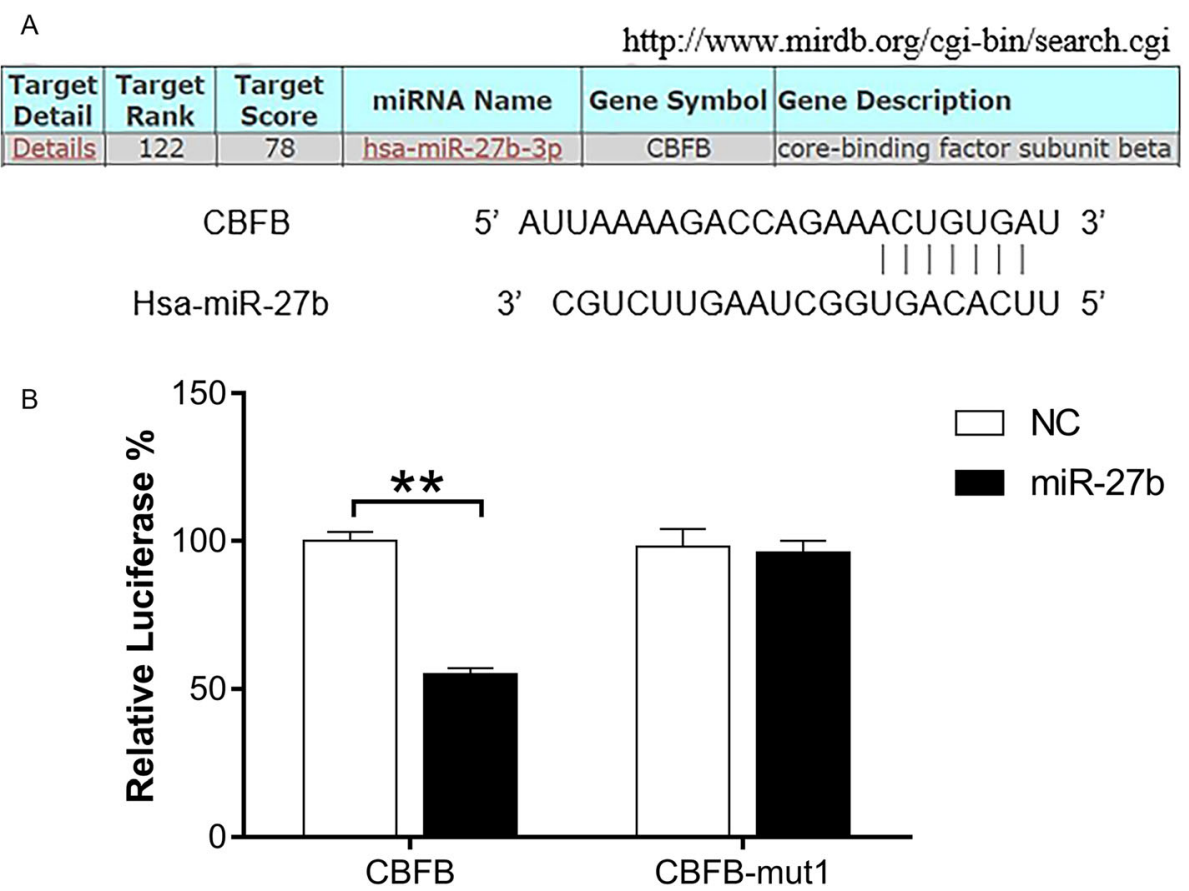

Fig. 6 CBFB is a novel target gene of miR-27b. a Predicted binding sites in the $3^{\prime}-U T R s$ of CBFB and miR-27b (microrna.org). $\mathbf{b}$ Luciferase activity of psiCHECK CBFB 3'-UTR. miR-27b distinctly suppressed the activity of the CBFB 3'-UTR, whereas CBFB-mut1 did not. **P<0.001 


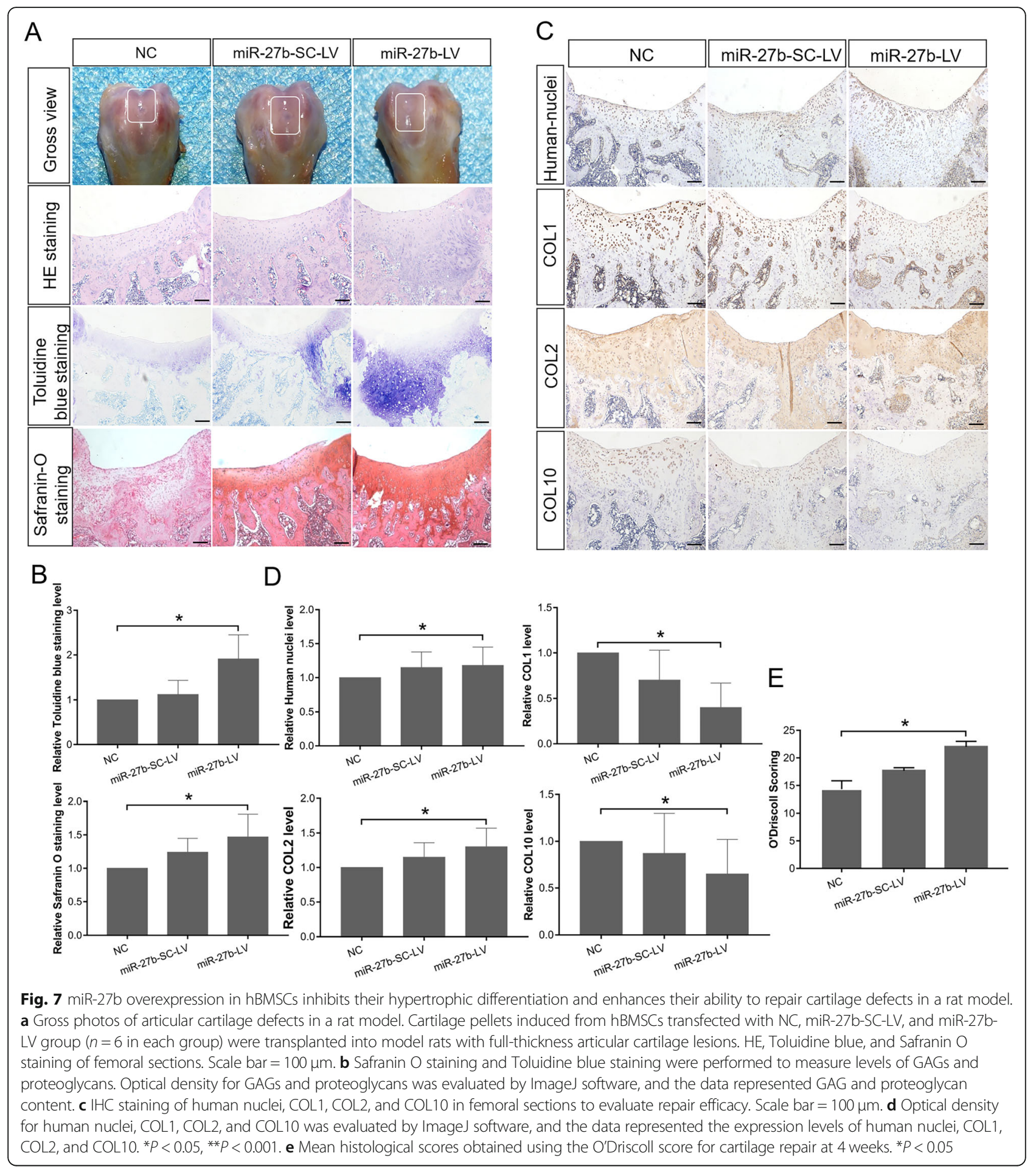

effectively alleviated hypertrophic chondrocyte differentiation and had tendency to enhance the repair efficacy of the hBMSCs in a rat cartilage injury model.

\section{Discussion}

In the present study, we investigated the expression profile of miR-27b and some of its biological functions during hBMSC chondrogenic differentiation. We made the following observations: (1) during hBMSC chondrogenic differentiation, miR-27b expression declines over time and is negatively related with hypertrophic differentiation, (2) CBFB is a novel direct target of miR-27b, (3) artificially elevating miR-27b levels in hBMSCs inhibits the phenotype in hBMSC chondrocytes, (4) within a 
cartilage defect environment in vivo, miR-27boverexpressing hBMSCs differentiate into nonhypertrophic chondrocytes and have improved repair efficacy compared with control hBMSCs. This study provided insights into the mechanism of hBMSC hypertrophic differentiation and new avenues for effective cartilage defect repair through hBMSC transplantation.

Accumulating evidence has shown that a number of miRNAs, including miR-140, miR-143, miR-145, miR483, miR-181a, miR-218, and miR-410, play critical roles in hBMSC chondrogenic differentiation $[15,23,25,40$, 44, 45]. The miR-140, miR-143, miR-145, miR-181a, and miR-221 expression patterns during hBMSC chondrogenic differentiation in our conditions were in line with those reported in previous studies [15]. Other investigators have reported that miR-145 target genes are involved in chondrocyte differentiation, including SOX9, $S M A D 4$, and $C B F B[20,46,47]$, and miR-140 target genes are SMAD1 and HDAC4 [39, 44]. During BMSC chondrogenic differentiation, miR-145 downregulation over time gradually reduces the inhibition of translation of its target genes. SOX9 is a master gene in the regulation of chondrogenesis and the maintenance of the chondrocyte phenotype. In contrast, SMAD4 and $C B F B$ are involved in hypertrophic differentiation of chondrocytes [48]. We found that at 14 days of pellet culture, despite maintaining high SOX9 mRNA expression, COL10, RUNX2, and CBFB expression levels were significantly enhanced, indicating that the cells had already begun hypertrophic differentiation. Through targeting SMAD1 and HDAC4, miR-140 inhibits hypertrophic differentiation [39, 44]. Strikingly, the miR-140 expression pattern seems to contradict this function. Combining our data with data from other groups, we speculate that during hypertrophic chondrocyte differentiation, the consistent upregulation of miR-140 expression, at least through inhibiting target gene translation, partially mitigates the progression to hypertrophy. Nevertheless, other mechanisms involving unidentified miRNAs or target genes downregulate protein expression and counteract this effect, allowing BMSC chondrogenesis to trend toward hypertrophy. Therefore, the miRNA expression pattern during cell differentiation provides important clues for further, more detailed and more functional investigation.

In the present study, we focused on miR-27b as a novel miRNA involved in BMSC hypertrophic chondrocyte differentiation. From 7 days post induction, the hypertrophic markers RUNX2 and COL10 were significantly upregulated. Furthermore, after artificially enhancing miR-27b expression in hBMSCs, the protein levels of both of SOX9 and COL2 were increased, whereas those of RUNX2 and COL10 level were decreased.
Combined with the in vivo data, these results indicate that low-level expression of miR-27b in hBMSCs during chondrogenic differentiation may contribute to hypertrophic differentiation. Consistent with that hypothesis, a previous study showed that during chondrogenic differentiation of hBMSCs, miR-27b expression tends to be upregulated. While no previous study has compared miR-27b expression between hBMSC-derived hypertrophic and normal chondrocytes, miR-27b has been reported as a key regulatory factor in chondrocyte development and differentiation [49]. We previously reported that in rat knee hyaline cartilage, chondrocytes in the superficial zone of articular cartilage had higher expression of miR-27b and miR-27a than did those in the hypertrophic zone. Further, we observed that when miR$27 \mathrm{~b}$ is overexpressed in hypertrophic chondrocytes, the hypertrophy marker COL10 protein level declined, and inversely, SOX9 and COL2 protein levels increased [26]. Consistent with those observations, in superficial zone cartilage of human limbs, miR-27 levels were approximately 2-3 times higher than in hypertrophic zone cartilage [50]. Based on these and our findings, we suggest that maintaining a high level of miR-27b in chondrocytes, including those derived from hBMSCs, is essential for inhibiting hypertrophic differentiation and maintaining the resting status of chondrocytes.

Like other miRNAs, miR-27b has multiple target genes, including MMP13, PPAR $2, M E F 2 C, S P 1, P H B$, and $P A X 3[26,27,49,51-55]$. The identification of target genes of miR-27b involved in hBMSC chondrocyte differentiation is significant for overcoming hypertrophic differentiation. In this study, we observed that during hBMSC chondrogenic differentiation, RUNX2 and COL10 showed similar gene expression patterns. Based on previous reports $[14,32]$, we hypothesized that if RUNX2 expression could be downregulated, hypertrophic differentiation would be attenuated. Consistent with those data, after miR-27b overexpression, the expression of RUNX2 protein significantly decreased. However, we found no putative miR-27b binding site in the 3'-UTR of RUNX2 mRNA. Therefore, we hypothesize that miR-27b indirectly represses RUNX2 protein expression. As is well known, CBFB cooperates with RUNX2 to form DNA-protein complexes and protects RUNX2 from degradation [29, 32]. Hence, we analyzed $C B F B$ as a potential target gene of miR-27b. Based on target prediction, western blot, luciferase reporter, and co-IP analyses, we validated that $C B F B$ is a direct target of miR-27b and indeed associates with RUNX2. Based on these results, we speculate that the increased level of miR-27b during differentiation effectively downregulates CBFB protein expression, leading to RUNX2 degradation and reduced RUNX2-DNA complex formation, resulting in the suppression of downstream gene 
expression. $M E F 2 C$ [52] and $P P A R \gamma$ [56], which also play key roles in regulating hypertrophic chondrocyte differentiation, are both miR-27b targets. Although we did not determine the effect of miR-27b on MEF2C protein expression, we suggest that artificially enhanced miR27b levels in hBMSCs may inhibit hypertrophic differentiation at least in part through simultaneously targeting $M E F 2 C, P P A R \gamma$, and CBFB mRNAs.

SOX9 is a master transcription factor that is essential for chondrogenic differentiation and development from MSCs [57]. Its expression is high in mitotic and early prehypertrophic chondrocytes, but downregulated in hypertrophic chondrocytes [42, 58]. In this study, we confirmed that during the 21-day chondrogenic differentiation, SOX9 expression was significantly upregulated at day 7 and was maintained at a high level, with an expression pattern similar to that of COL2. These data suggest that SOX9 is required for the initiation and promotion of chondrogenic differentiation of hBMSCs. SOX9 directly regulates chondrocyte-specific proteins, including COL2, aggrecan, and related proteins [59]. Moreover, SOX9 and RUNX2 directly interact through their evolutionarily conserved high-mobility group and runt domains, and SOX9 represses RUNX2 activity [60]. We observed that upon increasing miR-27b expression in hBMSCs, SOX9 and COL2 levels were significantly upregulated, whereas RUNX2 and COL10 expression was suppressed, indicating that hypertrophic differentiation may additionally be blocked by SOX9 overactivation or suppression of RUNX2 action.

Multiple signaling pathways have been shown to affect SOX9 expression in chondrogenic differentiation and development, including Hedgehog, bone morphogenetic protein, FGF, Notch, and WNT [61, 62]. Additionally, SOX9 expression in chondrocytes is strictly controlled by other factors (e.g., HIF1 $\alpha$, miR-145, SOX5) [63-65]. Wnt signaling plays an important role during chondrogenesis and hypertrophic differentiation [43]. Multiple studies have shown that the genes encoding the Wnt signaling regulatory components GSK-3 $\beta$, adenomatous polyposis coli, and Wnt3a are direct targets of miR-27b [66-68]. In addition, $\beta$-catenin expression is modulated by miR-27b [68], and in intestinal epithelium cells, $\beta$-catenin/TCF4 is required for SOX9 expression [69]. However, these studies on the relationship between miR-27b and Wnt signaling used cancer cell lines or normal cells other than hBMSCs. Here, we proved that miR-27b modulates SOX9 expression through regulating $\beta$-catenin expression during hBMSC chondrogenic differentiation. Given that SOX9 inhibits Wnt signaling by promoting $\beta$-catenin phosphorylation [70], there may exist a feedback loop between SOX9 and $\beta$-catenin. Future studies will need to investigate the detailed relationship between SOX9 and $\beta$-catenin after miR-27b treatment during hBMSC chondrogenic differentiation.
MicroRNAs have emerged as potent therapeutic targets in the treatment of multiple diseases and injuries. To effectively improve intercellular miRNA levels and enhance their therapeutic efficacy, multiple exogenous microRNA delivery systems have been developed, including lentiviral and adenoviral vectors [71], poly (lactide-co-glycolide) [72], and liposomes [73]. In this study, we selected lentiviruses as our vector for delivering exogenous miR-27b. Current lentiviral and adenoviral vectors all have limitations, including safety issues and loss of miRNA efficiency, which seriously impede the clinical application of miRNAs [74]. Therefore, safer and more efficient viral vectors have to be developed.

This study provides new insights into the mechanism of hBMSC chondrocyte differentiation that will aid in the development of novel strategies for the treatment of cartilage injury based on the transplantation of hBMSCs expressing high levels of miR-27b. However, it has some limitations. First, we only focused on a single miR-27b target gene, $C B F B$, to elucidate the mechanism through which miR-27b modulates hypertrophic differentiation. MicroRNAs have numerous target genes and modulate multiple signaling pathways. Therefore, the detection of ectopic miR-27b overexpression in hBMSCs through high-throughput mRNA and/or proteomic analyses will provide more detailed and comprehensive data for elucidating the functions and mechanisms of miR-27b. Second, we utilized the rat cartilage defect model, in which the injured cartilage environment completely varies from the pathology of OA; this difference may be a leading cause of hypertrophic chondrocyte differentiation in hBMSCs. Accordingly, another suitable animal OA model is required to transplant the hBMSCs overexpressing miR-27b into the OA cartilage defect site to investigate neocartilage properties and therapeutic efficiency. After conducting detailed mechanistic and preclinical animal studies, we can anticipate that miR27b-expressing hBMSC transplantation will be an effective clinical therapy for cartilage injury, including OA.

\section{Conclusion}

Maintaining high-level miR-27b expression in hBMSCs is necessary for inhibiting hypertrophic chondrogenesis and is a potential therapeutic option for articular cartilage repair and preventing OA defects.

\section{Supplementary information}

Supplementary information accompanies this paper at https://doi.org/10. 1186/s13287-020-01909-y.

Additional file 1: Fig S1. hBMSCs multidifferentiation potential. (A) Differentiation into osteoblasts as verified by alizarin red staining. After 4 weeks of induction, calcium nodules were observed. Scale bar $=100 \mu \mathrm{m}$. (B) Differentiation into adipocytes verified by Oil Red O staining. After 3 weeks of induction, intracellular lipid droplets were detected. Scale bar = 
$50 \mu \mathrm{m}$. (C) HE staining. Scale bar $=100 \mu \mathrm{m}$. (D) Alcian blue staining. Scale $\mathrm{bar}=50 \mu \mathrm{m}$. (E) Safranin O staining. Scale bar $=50 \mu \mathrm{m}$.

Additional file 2: Fig S2. miR-27b unregulated SOX9 expression through $\beta$-catenin. (A) Protein levels of $\beta$-catenin and SOX9 in hBMSCs after being transfected with miR-27b mimic and scramble as measured by western blot. (B) Semi-quantification of western blot data. ${ }^{*} P<0.05$, ${ }^{*} P<0.001$. (C) Protein levels of $\beta$-catenin and SOX9 in hBMSCs transfected with miR-27b mimic, si-SC and si- $\beta$-catenin as measured by western blot. (D) Semi-quantification of western blot data. ${ }^{*} P<0.05$, ${ }^{* *} P<0.001$.

Additional file 3. The prediction results of miR-27b binding site to 3 'UTR of CBFB MRNA.

Additional file 4. $I \mathrm{HC}$ staining of OPN and OCN for detecting endochondral bone formation in the hypertrophic cartilage region. Positive control is growth plate area in the same slide. Scale bar $=50 \mu \mathrm{m}$. The blank arrow denotes bone marrow cavity structures, and white arrow denotes the junction area between the hypertrophic zone of cartilage and subchondral bone. The black triangle denotes hypertrophic zone of cartilage in the positive control.

\section{Abbreviations}

BMSCs: Bone marrow mesenchymal stem cells; OA: Osteoarthritis; miR27b: microRNA-27b; COL: Collagen; MMP13: Matrix metalloproteinase 13; IHH: Indian hedgehog; miRNAs: microRNAs; LncRNAs: Long noncoding RNAs; UTR: Untranslated region; HDCA4: Histone deacetylase 4; PPARY: Peroxisome proliferator-activated receptor $\gamma$; CBFB: Core-binding factor, $\beta$-subunit: RUNX1-3: Runt-related transcription factors 1-3; FBS: Fetal bovine serum; bFGF: Basic fibroblast growth factor; EDTA: Ethylenediaminetetraacetic acid; PBS: Phosphate-buffered saline; TGF- $\beta 3$ : Transforming growth factor $\beta 3$; NC: Negative control; co-IP: Coimmunoprecipitation; miR-27b-SC-LV: miR27b-scramble-LV; HE: Hematoxylin and eosin; GAG: Glycosaminoglycan; IHC: Immunohistochemistry; chon: Chondrocytes; OPN: Osteopontin; OCN: Osteocalcin

\section{Acknowledgements}

We thank Deying Wang at The First Hospital of Jilin University for material assistance.

\section{Authors' contributions}

The study was designed by GC and YL. SL carried out most of the experiments, performed the statistical analyses, and drafted the manuscript. $J X, L C, W F, H Z$, and $L Z$ carried out some of the experiments. JX and $Y Z$ helped with the statistical analyses. PL and HW helped with the editing of the paper. All authors have read and approved the final manuscript.

\section{Funding}

This work was supported by the China Natural National Science Foundation (81970547, 81571199), the Jilin Province Science and Technology Development Plan (20190304039YY).

\section{Availability of data and materials}

All data generated or analyzed during this study are included in this published article.

\section{Ethics approval and consent to participate}

The primary human BMSC experiments were approved by the first hospital of Jilin University, Jilin University Ethics Committee (2017-342). Cells were collected from donors with written informed consent in accordance with the guidelines of the ethics committee of the Jilin University Hospital. Animal experiments were approved by the Ethical Committee of the School of Basic Medical Science, Jilin University Ethics Committee (2019064). Methods for each procedure were performed in accordance with the approved guidelines and regulations.

\section{Consent for publication}

Not applicable.

\section{Competing interests}

The authors declare that they have no competing interests.

\section{Author details}

${ }^{1}$ The Key Laboratory of Pathobiology, Ministry of Education, Department of Pathology, College of Basic Medical Sciences, Jilin University, Changchun 130021, China. ${ }^{2}$ Department of Gastrointestinal Surgery, Sino-Japanese Friendship Hospital of Jilin University, Changchun 130021, China. ${ }^{3}$ Department of Oncology, the First Hospital of Jilin University, Changchun 130021, China. ${ }^{4}$ Department of Bone and Joint, the First Hospital of Jilin University, Changchun 130021, China.

Received: 24 April 2020 Revised: 9 August 2020 Accepted: 27 August 2020 Published online: 11 September 2020

\section{References}

1. Yamashita A, et al. Generation of scaffoldless hyaline cartilaginous tissue from human iPSCs. Stem Cell Rep. 2015;4(3):404-18.

2. Heidari B. Knee osteoarthritis prevalence, risk factors, pathogenesis and features: part I. Caspian J Intern Med. 2011;2(2):205-12.

3. Fuggle NR, et al. Alternative and complementary therapies in osteoarthritis and cartilage repair. Aging Clin Exp Res. 2020;32(4):547-60.

4. Driessen BJH, Logie C, Vonk LA. Cellular reprogramming for clinical cartilage repair. Cell Biol Toxicol. 2017;33(4):329-49.

5. Mundi $\mathrm{R}$, et al. Cartilage restoration of the knee: a systematic review and meta-analysis of level 1 studies. Am J Sports Med. 2016;44(7):1888-95.

6. Mithoefer $\mathrm{K}$, et al. Clinical efficacy of the microfracture technique for articular cartilage repair in the knee: an evidence-based systematic analysis. Am J Sports Med. 2009;37(10):2053-63.

7. Kwon $\mathrm{H}$, et al. Surgical and tissue engineering strategies for articular cartilage and meniscus repair. Nat Rev Rheumatol. 2019;15(9):550-70.

8. Lee $\mathrm{HL}$, et al. Transforming growth factor- $\beta$-induced KDM4B promotes chondrogenic differentiation of human mesenchymal stem cells. Stem Cells. 2016;34(3):711-9.

9. Koga $\mathrm{H}$, et al. Mesenchymal stem cell-based therapy for cartilage repair: a review. Knee Surg Sports Traumatol Arthrosc. 2009;17(11):1289-97.

10. Mwale $F$, et al. Limitations of using aggrecan and type $X$ collagen as markers of chondrogenesis in mesenchymal stem cell differentiation. J Orthop Res. 2006;24(8):1791-8.

11. Little $C B$, et al. Matrix metalloproteinase 13-deficient mice are resistant to osteoarthritic cartilage erosion but not chondrocyte hypertrophy or osteophyte development. Arthritis Rheum. 2009;60(12):3723-33.

12. Goldring MB, Tsuchimochi K, ljiri K. The control of chondrogenesis. J Cell Biochem. 2006:97(1):33-44.

13. Diederichs S, et al. Regulation of WNT5A and WNT11 during MSC in vitro chondrogenesis: WNT inhibition lowers BMP and hedgehog activity, and reduces hypertrophy. Cell Mol Life Sci. 2019;76(19):3875-89.

14. Liu CF, et al. Transcriptional control of chondrocyte specification and differentiation. Semin Cell Dev Biol. 2017:62:34-49.

15. Gabler J, et al. Stage-specific miRs in chondrocyte maturation: differentiation-dependent and hypertrophy-related miR clusters and the miR-181 family. Tissue Eng A. 2015;21(23-24):2840-51.

16. Cao $Z$, et al. Long noncoding RNA expression profiles in chondrogenic and hypertrophic differentiation of mouse mesenchymal stem cells. Funct Integr Genomics. 2017;17(6):739-49.

17. Martin EC, et al. MicroRNA regulation of stem cell differentiation and diseases of the bone and adipose tissue: perspectives on miRNA biogenesis and cellular transcriptome. Biochimie. 2016:124:98-111.

18. Endisha $\mathrm{H}$, et al. The complex landscape of microRNAs in articular cartilage: Biology, pathology, and therapeutic targets. JCI Insight. 2018;3(17):e121630.

19. Zhao C, et al. MicroRNA-29b regulates hypertrophy of murine mesenchymal stem cells induced toward chondrogenesis. J Cell Biochem. 2019;120:8742-53.

20. Yang $B$, et al. MicroRNA-145 regulates chondrogenic differentiation of mesenchymal stem cells by targeting Sox9. PLoS One. 2011;6(7):e21679.

21. Lee $\mathrm{S}$, et al. microRNA-495 inhibits chondrogenic differentiation in human mesenchymal stem cells by targeting Sox9. Stem Cells Dev. 2014;23(15): $1798-808$.

22. Mao G, et al. MicroRNA-92a-3p regulates the expression of cartilage-specific genes by directly targeting histone deacetylase 2 in chondrogenesis and degradation. Osteoarthr Cartil. 2017;25(4):521-32.

23. Anderson BA, McAlinden A. miR-483 targets SMAD4 to suppress chondrogenic differentiation of human mesenchymal stem cells. J Orthop Res. 2017;35(11):2369-77. 
24. Lee JM, et al. miR-892b inhibits hypertrophy by targeting KLF10 in the chondrogenesis of mesenchymal stem cells. Mol Ther Nucleic Acids. 2019; 17:310-22.

25. Chen $\mathrm{S}$, et al. MicroRNA-218 promotes early chondrogenesis of mesenchymal stem cells and inhibits later chondrocyte maturation. BMC Biotechnol. 2019;19(1):6.

26. $\mathrm{Xu}$ J, et al. miR-27b promotes type II collagen expression by targetting peroxisome proliferator-activated receptor- $\gamma 2$ during rat articular chondrocyte differentiation. Biosci Rep. 2018;38(1):BSR20171109.

27. Akhtar $\mathrm{N}$, et al. MicroRNA-27b regulates the expression of matrix metalloproteinase 13 in human osteoarthritis chondrocytes. Arthritis Rheum. 2010;62(5):1361-71.

28. Wu M, et al. Cbf $\beta$ governs osteoblast-adipocyte lineage commitment through enhancing $\beta$-catenin signaling and suppressing adipogenesis gene expression. Proc Natl Acad Sci U S A. 2017;114(38):10119-24.

29. Tian $\mathrm{F}$, et al. Core binding factor beta $(\mathrm{Cbf} \beta)$ controls the balance of chondrocyte proliferation and differentiation by upregulating Indian hedgehog (Ihh) expression and inhibiting parathyroid hormone-related protein receptor (PPR) expression in postnatal cartilage and bone formation. J Bone Miner Res. 2014;29(7):1564-74.

30. Wu $\mathrm{M}$, et al. Deletion of core-binding factor $\beta(\mathrm{Cbf} \beta)$ in mesenchymal progenitor cells provides new insights into $\mathrm{Cbf} \beta /$ Runxs complex function in cartilage and bone development. Bone. 2014;65:49-59.

31. Park NR, et al. Core binding factor beta plays a critical role during chondrocyte differentiation. J Cell Physiol. 2016;231(1):162-71.

32. Komori TK. Runx2, an inducer of osteoblast and chondrocyte differentiation. Histochem Cell Biol. 2018;149(4):313-23.

33. Xiao $D$, et al. Notch signaling regulates MMP-13 expression via Runx2 in chondrocytes. Sci Rep. 2019;9(1):15596.

34. Osiecka-Iwan A, Hyc A, Moskalewski S. Immunosuppression and rejection of cartilage formed by allogeneic chondrocytes in rats. Cell Transplant. 1999; 8(6):627-36.

35. Schmitz $\mathrm{N}$, et al. Basic methods in histopathology of joint tissues. Osteoarthr Cartil. 2010;18(Suppl 3):S113-6.

36. O'Driscoll SW, Keeley FW, Salter RB. Durability of regenerated articular cartilage produced by free autogenous periosteal grafts in major full-thickness defects in joint surfaces under the influence of continuous passive motion. A follow-up report at one year. J Bone Joint Surg Am Volume. 1988:70(4):595-606.

37. Haq SH. 5-Aza-2'-deoxycytidine acts as a modulator of chondrocyte hypertrophy and maturation in chick caudal region chondrocytes in culture. Anat Cell Biol. 2016;49(2):107-15.

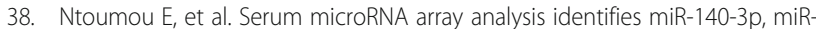
33b-3p and miR-671-3p as potential osteoarthritis biomarkers involved in metabolic processes. Clin Epigenet. 2017;9:127.

39. Papaioannou $\mathrm{G}$, et al. MicroRNA-140 provides robustness to the regulation of hypertrophic chondrocyte differentiation by the PTHrP-HDAC4 pathway. J Bone Miner Res. 2015;30(6):1044-52.

40. Tian J, et al. MiR-143-3p regulates early cartilage differentiation of BMSCs and promotes cartilage damage repair through targeting BMPR2. Eur Rev Med Pharmacol Sci. 2018;22(24):8814-21.

41. Komori T. Molecular mechanism of Runx2-dependent bone development. Mol Cells. 2020;43(2):168-75.

42. Hussain S, et al. SFMBT2 positively regulates SOX9 and chondrocyte proliferation. Int J Mol Med. 2018;42(6):3503-12.

43. Held A, et al. Targeting beta-catenin dependent Wnt signaling via peptidomimetic inhibitors in murine chondrocytes and OA cartilage. Osteoarthr Cartil. 2018;26(6):818-23.

44. Li C, et al. MicroRNA-140 suppresses human chondrocytes hypertrophy by targeting SMAD1 and controlling the bone morphogenetic protein pathway in osteoarthritis. Am J Med Sci. 2018;355(5):477-87.

45. Zhang $Y$, Huang $X$, Yuan Y. MicroRNA-410 promotes chondrogenic differentiation of human bone marrow mesenchymal stem cells through down-regulating Wnt3a. Am J Transl Res. 2017:9(1):136-45.

46. Zhou J, et al. MicroRNA-145-5p regulates the proliferation of epithelial ovarian cancer cells via targeting SMAD4. J Ovarian Res. 2020;13(1):54.

47. Fukuda T, et al. MicroRNA-145 regulates osteoblastic differentiation by targeting the transcription factor Cbfb. FEBS Lett. 2015;589(21):3302-8

48. Yan J, et al. Smad4 deficiency impairs chondrocyte hypertrophy via the Runx2 transcription factor in mouse skeletal development. J Biol Chem. 2018;293(24):916275.

49. Kara N, et al. miR-27 regulates chondrogenesis by suppressing focal adhesion kinase during pharyngeal arch development. Dev Biol. 2017;429(1): 321-34.
50. McAlinden A, et al. Differentially expressed microRNAs in chondrocytes from distinct regions of developing human cartilage. PLoS One. 2013;8(9):e75012.

51. Karbiener M, et al. MicroRNA miR-27b impairs human adipocyte differentiation and targets PPARgamma. Biochem Biophys Res Commun. 2009;390(2):247-51.

52. Chinchilla $A$, et al. MicroRNA profiling during mouse ventricular maturation: a role for miR-27 modulating Mef2c expression. Cardiovasc Res. 2011;89(1):98-108.

53. Jiang J, et al. MicroRNA-27b suppresses growth and invasion of NSCLC cells by targeting Sp1. Tumour Biol. 2014;35(10):10019-23.

54. Crist CG, et al. Muscle stem cell behavior is modified by microRNA-27 regulation of Pax3 expression. Proc Natl Acad Sci U S A. 2009;106(32):13383-7.

55. Kang T, et al. MicroRNA-27 (miR-27) targets prohibitin and impairs adipocyte differentiation and mitochondrial function in human adipose-derived stem cells. J Biol Chem. 2013;288(48):34394-402.

56. Monemdjou $\mathrm{R}$, et al. Association of cartilage-specific deletion of peroxisome proliferator-activated receptor $\gamma$ with abnormal endochondral ossification and impaired cartilage growth and development in a murine model. Arthritis Rheum. 2012;64(5):1551-61.

57. Jiang $X$, et al. Correction: the role of Sox9 in collagen hydrogel-mediated chondrogenic differentiation of adult mesenchymal stem cells (MSCs). Biomater Sci. 2019;7(9):3926.

58. Ohba S, et al. Distinct transcriptional programs underlie Sox9 regulation of the mammalian chondrocyte. Cell Rep. 2015;12(2):229-43.

59. Lefebvre V, Dvir-Ginzberg M. SOX9 and the many facets of its regulation in the chondrocyte lineage. Connect Tissue Res. 2017;58(1):2-14.

60. Zhou G, et al. Dominance of SOX9 function over RUNX2 during skeletogenesis. Proc Natl Acad Sci U S A. 2006;103(50):19004-9.

61. Turner BRH, Itasaki N. Local modulation of the Wnt/B-catenin and bone morphogenic protein (BMP) pathways recapitulates rib defects analogous to cerebro-costo-mandibular syndrome. J Anat. 2020;236(5):931-45.

62. Correa D, et al. Sequential exposure to fibroblast growth factors (FGF) 2, 9 and 18 enhances hMSC chondrogenic differentiation. Osteoarthr Cartil. 2015;23(3):443-53.

63. $\mathrm{Li} \mathrm{H}$, et al. Hypoxia promotes maintenance of the chondrogenic phenotype in rat growth plate chondrocytes through the HIF-1alpha/YAP signaling pathway. Int J Mol Med. 2018;42(6):3181-92

64. Martinez-Sanchez A, Dudek KA, Murphy CL. Regulation of human chondrocyte function through direct inhibition of cartilage master regulator SOX9 by microRNA-145 (miRNA-145). J Biol Chem. 2012;287(2):916-24.

65. Park JS, et al. Construction of PLGA nanoparticles coated with polycistronic SOX5, SOX6, and SOX9 genes for chondrogenesis of human mesenchymal stem cells. ACS Appl Mater Interfaces. 2017;9(2):1361-72.

66. Yu J, et al. CDX2 inhibits the proliferation and tumor formation of colon cancer cells by suppressing Wnt/B-catenin signaling via transactivation of GSK-3 3 and Axin2 expression. Cell Death Dis. 2019;10(1):26.

67. Aghabozorgi AS, et al. Role of adenomatous polyposis coli (APC) gene mutations in the pathogenesis of colorectal cancer; current status and perspectives. Biochimie. 2019;157:64-71

68. Lv $\mathrm{X}$, et al. Overexpression of miR-27b-3p targeting Wnt3a regulates the signaling pathway of Wnt/beta-catenin and attenuates atrial fibrosis in rats with atrial fibrillation. Oxidative Med Cell Longev. 2019;2019:5703764.

69. Blache $P$, et al. SOX9 is an intestine crypt transcription factor, is regulated by the Wnt pathway, and represses the CDX2 and MUC2 genes. J Cell Biol. 2004;166(1):37-47.

70. Topol L, et al. Sox9 inhibits Wnt signaling by promoting beta-catenin phosphorylation in the nucleus. J Biol Chem. 2009;284(5):3323-33.

71. Weissenberger $M$, et al. Reduced hypertrophy in vitro after chondrogenic differentiation of adult human mesenchymal stem cells following adenoviral SOX9 gene delivery. BMC Musculoskelet Disord. 2020;21(1):109.

72. Kunisch $E$, et al. The poly (I-lactid-co-glycolide; PLGA) fiber component of brushite-forming calcium phosphate cement induces the osteogenic differentiation of human adipose tissue-derived stem cells. Biomed Mater (Bristol, England). 2019;14(5):055012.

73. Boca $\mathrm{S}$, et al. Nanoscale delivery systems for microRNAs in cancer therapy. Cell Mol Life Sci. 2020;77(6):1059-86.

74. Grol MW, Lee BH. Gene therapy for repair and regeneration of bone and cartilage. Curr Opin Pharmacol. 2018;40:59-66.

\section{Publisher's Note}

Springer Nature remains neutral with regard to jurisdictional claims in published maps and institutional affiliations. 